\title{
Trekstock Meet \& Move: The impact of one- day health and well-being events for young adults with cancer
}

Pugh, Gemma ${ }^{1}$ PhD; Petrella, Anika ${ }^{4}$ MA; Fisher, Abigail ${ }^{3}$ PhD; Reynolds, Jemima ${ }^{2}$; Epstone,

Sophie ${ }^{2}$

1. Centre for Sports \& Exercise Medicine, Queen Mary University of London, London, United Kingdom

2. Trekstock, London, United Kingdom

3. Department of Behavioural Science \& Health, University College London, London, United Kingdom

4. Department of Exercise Sciences, University of Toronto, Toronto, Canada

g.pugh@qmul.ac.uk

anika.petrella@mail.utoronto.ca

abigail.fisher@ucl.ac.uk

sophie.epstone@trekstock.com

health@trekstock.com

Date: August 2019

Corresponding Author

Dr Gemma Pugh PhD

Centre for Sports \& Exercise Medicine

Mile End Hospital

London

E1 4DG

Email: g.pugh@qmul.ac.uk

Telephone: 02078825015

Running Head: Meet and Move for Young Adult Cancer Survivors

Key Words: Young Adult, Cancer, Physical Activity, Survivorship 


\section{Abstract}

Purpose: To evaluate the impact of a series of one-day events delivered by Trekstock a cancer charity supporting young adults with cancer in the United Kingdom.

Method: Data on physical activity, mood, perceived support, self-efficacy and confidence to be active were collected at three time points: before, after, and 2 weeks following the Meet \& Move event.

Results: Ninety-seven young adults with cancer (mean age: 29 years, 35\% still receiving active treatment) attended a Trekstock Meet \& Move event $(n=5)$ and participated within the evaluation. Baseline data demonstrated that prior to attending a Meet \& Move event $27.4 \%(n=23)$ of young adults reported felt their cancer excluded them from engaging in exercise, $44 \%(n=37)$ reported worry exercise will cause pain or injury and only $38.6 \%(n=32)$ knew what exercise they could do. Data collected post-event and at follow-up indicated that Meet \& Move had a positive impact upon attendees' self-efficacy and confidence to be active with more than half reporting they felt inspired after attending. There was also a significant reduction in reported worry that exercise may cause pain or injury and reported perception of feeling left out of exercise because of cancer $(p<0.005)$. Following engagement in the Meet \& Move events, $45 \%$ of attendees had either signed up for an additional Trekstock physical activity program or initiated engagement in a new type of physical activity on their own.

Conclusion: Trekstock Meet \& Move events inspire and motivate young adults with cancer in their 20 s and 30 s to be active. 


\section{Background}

It is estimated that 14,898 young adults in their 20's and 30's are diagnosed with cancer in each year in the United Kingdom [1]. Analyses of population-registry data from the United States indicate that this figure is set to rise, particularly for obesity-related cancers [2]. Thankfully, due to advances in the early detection and treatment approximately $80 \%$ of young adults in their 20 s and 30s diagnosed with cancer are expected to survive for 5 or more years [3]. The growing incidence of cancer and increase in survival for those aged 20-39 has led to an increase in the number of young adults requiring cancer support. In 2014, the Macmillan segmenting the million project estimated that there were approximately 200,000 young people in their late-20s and 30s classed as cancer survivors within the United Kingdom [4].

Given that cancer is a disease which most commonly affects those over the age of 60 most cancer services are designed to reflect the needs of older adults [5].Teenage and Young Adult (TYA) cancer care is well established within the UK for young people diagnosed between the age of 13 and 24 years of age. A young person diagnosed in this age bracket will receive treatment in a principal treatment centre or designated hospital by a specialized multidisciplinary team. Following treatment, young people will be transitioned to specialized long-term follow up or shared care between a TYA survivorship clinic and their general practitioner [6]. However, despite having similar needs young adults in their late twenties and thirties (age range 25-39 years) do not receive similar age-appropriate clinical care or support. This has resulted in young adults who do not fall within the traditional TYA age-bracket reporting poor psychosocial well-being including feelings of isolation, depression, and anxiety [7-9].

Trekstock, a cancer charity based in London United Kingdom was established to address this gap by providing emotional and practical support to young adults faced with cancer in their 20 s and 30s. Based upon substantial evidence that physical activity is safe, feasible and has a number of 
clinical, physical and psychosocial benefits for cancer patients both during and after treatment $[10,11]$; Trekstock focuses on helping young adults with cancer to be active following their diagnosis. Physical activity behaviour has been shown to be a predictor of psychosocial wellbeing among young adults with cancer [12] and has been recognised by young adults themselves as a means to regain control over their health and well-being [13]. However, less than $50 \%$ of young adults with cancer are active [14]. Recent data indicate self-efficacy, confidence, and knowledge are important predictors of exercise behaviour among young adults with cancer [15]. Interventions which aim to improve young adult cancer survivors' self-efficacy and confidence to be active through education and peer-support are therefore warranted.

Within the United Kingdom, a core recommended feature of the National Cancer Survivorship Initiative (NSCl) 'Recovery Package' is the inclusion of a health and wellbeing event containing support and information on life beyond cancer including going back to work, managing finances, coping with the long-term effects of treatment and making healthy lifestyle choices. As many existing wellbeing events were developed with older adults in mind, Trekstock developed a series of one day events titled 'Meet \& Move' to provide opportunities for young adults with cancer to meet with other cancer survivors of a similar age and receive age-appropriate advice on how to live well post cancer diagnosis and treatment. The purpose of this evaluation was to determine the impact of the Meet \& Move events on young adults' well-being, self-efficacy and motivation to exercise. Evaluation outcomes included understanding i) what motivates young adults with cancer to attend Meet \& Move events ii) if Meet \& Move events help address feelings of loneliness, isolation and worry in young people with cancer and iii) if attending a Meet \& Move events influences the self-efficacy and confidence of young people with cancer to be active. 


\section{Methods}

\section{Design}

Across 2018 and 2019 an evaluation of five Meet \& Move events was conducted using a pre and post-test design. Participants were asked to complete a self-report questionnaire before, immediately after, and 2 weeks following attending a Meet \& Move event. Self-report data on participants' levels of physical activity, mood, self-efficacy and confidence to exercise was collected alongside open-ended questions to gain young peoples' subjective viewpoint of the Meet \& Move events. Queen Mary, University of London Research Ethics Committee approved the collection of the data.

An overview of the programmes of each Meet \& Move events is contained within Appendix A. Each event was carried out at a Maggie's Cancer Support centre and consisted of i) opportunity to engage in physical activity through a group led exercise session and a yoga class ii) a food and nutrition workshop centered around a cooking demonstration and iii) sessions covering the psychosocial impact of cancer. Other activities on offer across the five events included art therapy, gardening, Look Good Feel Better skin and make up sessions and comedy. Meet \& Move events were free to attend for participants. The cost incurred by Trekstock per person to attend a Meet \& Move event was £95.66.

\section{Outcomes Measures}

\section{Demographics Questions}

Participants were asked to report their age, type of cancer diagnosis, age at diagnosis and current treatment status. 


\section{Physical Activity}

The Godin Leisure Time Exercise Questionnaire (GLTEQ) was used to assess physical activity at baseline and follow-up. Participants were asked to report the frequency of time they spent carrying out strenuous exercise (e.g. running, jogging, vigorous swimming), moderate exercise (e.g. fast walking, easy cycling, easy swimming) and mild exercise (e.g. easy walking, fishing and bowling). The GLTEQ has been used in previous studies of TYA cancer survivors $[14,16,17]$ and has demonstrated reliability and validity within the oncology research setting [18].

\section{Self-Efficacy \& Confidence}

The nine-item Self-Efficacy for Exercise (SEE) [19] scale was used to assess self-efficacy to exercise at each time-point. Participants were asked to rate on a scale of 1-5 their level of confidence to exercise for more than 10 minutes/exercise for more than 30 minutes/exercise alone/take part in a new form of exercise/exercise even if they were busy with new activities/ exercise if they felt tired/exercise if they felt stressed/and exercise if they felt depressed. Higher scores indicate greater confidence to overcome barriers to exercise. The SEE scale has previously been used in studies of cancer survivors [20,21] and has strong internal consistency (Alpha coefficient 0.92) [19]. Participants were also asked to rate on a 5 point 'Likert scale (1 being not at all, 5 being very much) the extent to which family and friends support them being physically active, if they felt cancer prevented them exercising, their knowledge of help and support to be active, the extent they worry about exercise causing pain or injury and whether they knew of exercises they were capable of doing. 
Mood

To assess mood, a single-item question whereby respondents rated if they felt (happy/hopeful/relaxed/anxious/lonely/troubled) both before and after the event was included within the post-event questionnaire.

\section{Motivation to attend and impact of Meet \& Move}

To understand what participants were hoping to gain from attending the Meet \& Move participants were asked to select from the following list what motivated them to attend: to try new physical activity options/ to kick start getting back into exercise after my cancer diagnosis/ because my friends/ family/partner said I should come/ I wanted to meet other young adults with cancer/ I wanted to find out more about Trekstock. In order to understand the benefit of the Meet \& Move event, participants were asked to select if they agreed (Yes/No) with the following statements: I feel inspired to be more active, l've picked up new ideas on how to be more active, I feel more confident to exercise regularly, I now know there are other young people like me who also struggle to stay active.

\section{Analysis}

SPSS Version 22 was used to perform statistical analyses. Descriptive data on participant characteristics, physical activity levels, motivation to attend and impact of Meet \& Move were reported as percentage, mean and standard deviation values. Changes in participants' levels of physical activity, self-efficacy and confidence were established using repeated measures (within subject) ANOVAs. To examine differences at baseline and follow-up paired t-tests were used for physical activity and Wilcoxon Signed Rank Test for mood. Standard GLTEQ scoring was used to calculate a Leisure Score Index (LSI) which was used to categorize participants as inactive or insufficiently active ((estimated energy expenditure $<14 \mathrm{Kcal} / \mathrm{kg} /$ week) [22]. 


\section{Results}

\section{$\underline{\text { Participant characteristics }}$}

Table1 displays participant characteristics. Ninety-seven young people with cancer attended Meet \& Move events in 2018 \& 2019 held in West London, East London, Manchester, Oxford and Newcastle. All participants completed the baseline questionnaire, 83 completed the post-event questionnaire and 24 completed the follow-up questionnaire 2 weeks after the event. Participants were between 18 and 43 years of age (mean age: $29 \pm 5.49$ years). Mean age at diagnosis was $26 \pm 7.34$ years). Most participants had been diagnosed with cancer during their 20 's ( $n=42,50 \%$ vs $n=30,35.7 \%$ diagnosed in their 30 s). 8 participants $(9.5 \%)$ were long-term survivors of a childhood or adolescent diagnosis (diagnosed before the age of 18). The most common cancer diagnoses was carcinoma $(n=37,44 \%)$ of whom $64 \%(n=24)$ reported breast cancer. The majority of participants were still receiving active cancer treatment $(35.1 \%, n=34)$ or were in the first year post treatment $(35.7 \%, n=35)$. Most young people $(55.7 \%, n=54)$ heard about the event online through Instagram, Twitter or the Trekstock website and came to the Meet \& Move event to meet other young people with cancer $(66 \%, n=64)$. Forty-one percent of young adults who attended Meet \& Move events were active $(n=40$ LSI Score $\geq 24$, Sample Mean $\pm S D=26.31 \pm 34.09$ ) and reported strong family and peer support to be active $(42.3 \%, n=40$ selecting very much so on a 5-point likert scale, Mean $\pm S D: 4 \pm 1.08$ ). At baseline prior to attending a Meet\&Move event $27.4 \%$ $(n=23)$ of young adults reported feeling left out of exercise because of their cancer, $44 \%(n=37)$ reported that they were worried exercise will cause pain or harm and only $38.6 \%(n=32)$ reported knowing what exercise they could do.

\section{The impact of Meet \& Move}

As shown in Figure 1 before attending a Meet \& Move event only $8 \%$ of young adults reported feeling happy with some reporting feeling anxious, lonely and troubled. Following attending the 
proportion of young people reporting feeling happy, hopeful or relaxed rose significantly $(p<0.005)$.

Figure 2 displays mean scores and change in self-reported rating of perceived support and selfefficacy to exercise. Following Meet \& Move, a significant difference in young adults feelings of being left out of exercise $(F(1,17)=4.33, p=0.053)$ and worry that exercise will cause pain or injury was observed $(F(1,18)=4.98, p=0.038)$. The Meet \& Move events also improved young adults knowledge about the types of exercise they can do $(F(1,16)=2.75, p=0.116)$ and where they can access help and support to be more active $(F(1,17)=4.86, p=0.495)$.

Following attending a Meet \& Move event $64 \%(n=51)$ agreed with the statement they now knew there are other young people like them with cancer who also struggle to stay active and $52 \%$ $(n=41)$ felt inspired to be active. Forty-seven percent $(n=37)$ picked up new ideas on how to be more active from attending and $32 \%(n=25)$ felt more confident to exercise regularly. The majority of young adults $(94 \%, n=78)$ said they would 'definitely' recommend the Meet \& Move event to another young adult living with cancer.

Table 3 displays mean scores and change in confidence regarding physical activity. Among young adults who attended the Meet \& Move events overall confidence to exercise was high. Although not statistically significant results suggest Meet \& Move improved participants' confidence to exercise for extended periods of time, take part in new forms of activity and exercise even when feeling low (e.g. tired, stressed or depressed).

Forty-five percent $(n=11)$ of young people who completed the follow-up questionnaire 2 weeks $(n=24)$ after the Meet \& Move event said they had either signed up to the RENEW exercise referral programme offered by Trekstock or had started another type of physical activity on their own. There was no significant difference in the reported activity levels of participants at two-week follow 
up in comparison to baseline $(p=0.087)$. Eighty-seven percent $(n=21)$ said they would be 'very likely' to attend the event again with the remaining $12.5 \%(n=5)$ reporting they would be 'likely' to attend again. Most young adults $(66 \%, n=16)$ said they would like to attend a Meet \& Move event every two months.

Written praise and suggestions for improvement from young adults who attended the Meet \& Move events are contained within Table 2. Although meeting other young people with cancer was one of the primary reasons young people attended and enjoyed the Meet \& Move event, one young person highlighted that meeting other people at a different stage in the cancer journey may be intimidating. Several young people also suggested name badges would be a useful addition to the day to make introductions and starting conversations easier. One young person noted that during the group coaching sessions there was a tendency for participants to share negative thoughts and experiences.

\section{Discussion}

This evaluation provides an insight into the benefit of one day health and wellbeing events designed specifically for young adults with cancer. Results demonstrate that Meet \& Move had a positive impact upon attendees self-efficacy and confidence to be active with more than half reporting they felt inspired after attending. There was also a significant reduction in reported worry that exercise may cause pain or injury and reported perception of feeling left out of exercise because of cancer. $63-76 \%$ of participants reported feeling happy, hopeful and relaxed at the end of the event highlighting the role peer support plays in improving young adults psychosocial wellbeing. Forty-five percent of participants who completed the follow-up questionnaire at 2 weeks had either signed up for other Trekstock physical activity offerings or had started another type of physical activity. 
Through meeting other young people with cancer Meet \& Move may help address feelings of isolation and fear surrounding being active following a cancer diagnosis. This was demonstrated by the large proportion of participants who acknowledged that the event increased their awareness of other young people with cancer who also struggle to stay active. The need for young people with cancer to communicate with other young people in a similar situation to them is well documented within the literature [5, 23], especially given that a cancer diagnosis during early adulthood is incredibly rare and family, friends, and caregivers may not have an understanding about the dormant or latent effects of cancer [24-26]. Within one study of 514 young adults diagnosed with cancer in Germany (mean age: 29 years) social support and positive interactions with others were found to be associated with life satisfaction after treatment completion during the first 12 months of survivorship. Awarding micro-grants to support women with breast cancer to develop and implement physical activity initiatives in their local area has proved effective in Canada [27]. A similar initiative or a buddy system partnering young people who are at a similar stage of treatment may be an effective way of ensuring Meet \& Move attendees can connect with peers who have had a similar cancer diagnosis or are at a similar stage of treatment.

A large proportion of participants reported that the event 'inspired' them to be more active and that they would definitely recommend attending. Post Meet \& Move and at follow-up, the number of young adults reporting feeling left out of exercise because of their cancer or not knowing what exercise to do fell significantly. Statistically significant improvements in self-efficacy and confidence to exercise were not found, however this find may be attributed to Meet \& Move attendees demonstrating high levels of physical activity assurance at baseline. Existing marketing strategies (such as linguistically and culturally tailoring advertisements) previously piloted among hard-to-reach populations $[28,29]$ should be tested to attempt to engage young adults with low levels of confidence and self-efficacy to attend Meet \& Move events. These individuals may reap greater benefit from Meet \& Move events than individuals who already exercise and are engaged 
with leading a healthy lifestyle. This also applies to those who have high levels of health anxiety or negative emotion towards supportive care interventions. A stepped-care approach, whereby those who are less engaged or who have specific needs receive tailored support, may be an effective means to widen the reach of health promotion initiatives for cancer survivors [30].

A range of young adults with a variety of cancer diagnoses and at various different stages of treatment attended Meet \& Move showing that the programme of events appeals to young adults at different stages of the cancer journey. However, the majority of young adults heard about the events via Facebook, Twitter or Instagram. Resources and health promotion materials only available in digital formats require proactive information seeking which is linked to greater levels of health engagement and higher levels of health literacy [31]. It is likely that Trekstock followers on Facebook, Twitter and Instagram are engaged and motivated to change their health behaviour. Health professionals (inclusive of doctors, nurses, social workers and members of the allied health professions) have been identified as key gate-keepers to pathways which connect cancer patients with exercise-related resources [32]. Increasing awareness about Meet \& Move among health professionals working in young adult cancer care and providing them with physical resources to sign-post young people towards the online registration for Meet \& Move is a potential solution to engaging more young adults with cancer with Meet \& Move events.

This brief evaluation demonstrates the Meet \& Move is well received by, and has a positive impact upon young people with cancer. However, the evaluation is somewhat limited by the lack of control group. In order to reduce participant burden it was felt that each questionnaire should be twopages long. Although a two-week follow up questionnaire was included within the evaluation a number of participants did not respond or complete this. Future work should aim to explore strategies which encourage young adults with low motivation to change their physical activity behaviour to attend Meet and Move events and strategies which sustain positive lifestyle behaviour change long-term. The generalisability of the results are also somewhat limited as the 
sample predominantly consists of female breast cancer survivors who are highly educated and already active. This is a common problem observed in exercise oncology research. In acknowledgment of the findings Trekstock, in partnership with academics from Queen Mary University of London, have carried out a series of public involvement and engagement activities to address how the reach of the Meet \& Move initiative can be expanded, specifically towards men.

\section{Conclusion}

As per the National Cancer Survivorship Initiative (NCSI), all cancer patients should have access to an education and support event as part of the cancer recovery package. NCSI guidance suggests these events facilitate self-management through the provision of education and support on the physical activity and lifestyle behaviour change. The Meet \& Move event provided by Trekstock offers an age -specific alternative for young people who fall outwith of the traditional TYA age bracket in the United Kingdom. To expand the success of Meet \& Move events Trekstock are considering existing recovery package resources currently commissioned within the NHS and pathways by which Meet \& Move events could be integrated into routine health and social care for young people with cancer.

\section{Acknowledgments}

The authors acknowledge the support of the Maggie's Centres charity in supporting the delivery of Meet \& Move events. Specifically, Liz Shaladan, Maggie's Centres Operation Manager for the South of England and the Maggie's Centre Heads at West London, Barts, Oxford, Manchester and Newcastle. A thank you and special acknowledgement to Toby Peach, Chevy Rough, Liz O'R Riordan, Steph Davies, Teresa Waite, Joelle Rainford, Poopy Stevens, Hannah Niven, Robynne Cranston, Grace Cooper and Dani Binnington for contributing towards the delivery of the Meet \& Move events. 


\section{Disclaimer}

All data was collected by JR and SE; GP analysed and drafted the manuscript; AF and AP provided comments and input upon the manuscript.

\section{Author Disclosure Statement}

SE is the Chief Executive Officer of Trekstock; JR is employed by Trekstock; GP has received remuneration for providing independent consultancy to Trekstock. No competing financial interests exist for AF or AP. 


\section{References}

1. Cancer, I.A.f.R.o. Data visualization tools for exploring the global cancer burden in 2018 2019 [cited 2019 July].

2. Sung, H., et al., Emerging cancer trends among young adults in the USA: analysis of a population-based cancer registry. The Lancet Public Health, 2019. 4(3): p. e137-e147.

3. O'Hara, C., et al., Trends in survival for teenagers and young adults with cancer in the UK 1992-2006. European Journal of Cancer, 2015. 51(14): p. 2039-2048.

4. NCIN, N.C.I.N., Macmillan-NCIN work plan. Segmenting the cancer survivor population by cancer type, 20 year prevalence at the end of 2010, UK. . 2013.

5. Barr, R.D., et al., Cancer in adolescents and young adults: A narrative review of the current status and a view of the future. JAMA Pediatrics, 2016. 170(5): p. 495-501.

6. Teenage Cancer Trust, <Blueprint of Care. Teenage Cancer Trust..pdf>. 2012.

7. Aubin, S., et al., Cancer!? I Don't Have Time for That: Impact of a Psychosocial Intervention for Young Adults with Cancer. Journal of Adolescent and Young Adult Oncology, 2019. 8(2): p. 172-189.

8. Geue, K., et al., Prevalence of mental disorders and psychosocial distress in German adolescent and young adult cancer patients (AYA). Psycho-Oncology, 2018. 27(7): p. 1802-1809.

9. Lang, M.J., et al., Does age matter? Comparing post-treatment psychosocial outcomes in young adult and older adult cancer survivors with their cancer-free peers. PsychoOncology, 2018. 27(5): p. 1404-1411. 
10. McTiernan, A., et al., Physical Activity in Cancer Prevention and Survival: A Systematic Review. Medicine and Science in Sports and Exercise, 2019. 51(6): p. 1252-1261.

11. Sabiston, C.M. and J. Brunet, Reviewing the benefits of physical activity during cancer survivorship. Am J Lifestyle Med, 2012. 6.

12. Wurz, A. and J. Brunet, Does physical activity moderate the associations between body mass index and self-perceptions in adolescent and young adult cancer survivors? Journal of Sport \& Exercise Psychology, 2018. 40: p. S125-S125.

13. Pugh, G., et al., The Lifestyle Information and Intervention Preferences of Teenage and Young Adult Cancer Survivors: A Qualitative Study. Cancer Nurs, 2017.

14. Pugh, G., et al., The health behaviour status of teenage and young adult cancer patients and survivors in the United Kingdom. Support Care Cancer, 2019.

15. Diorio, C.M., L.; Ginn, E.; Ladas, E Psychosocial determinants of physical activity and dietary behaviors in adolescents and young adults with cancer and survivors. Pediatric Blood \& Cancer 2018 Online Version of Record before inclusion in an issue

\section{e27243.}

16. Wright, M., et al., Physical Activity in Adolescents following Treatment for Cancer: Influencing Factors. Leuk Res Treatment, 2013. 2013: p. 592395.

17. Gilliam, M.B., et al., Social, Demographic, and Medical Influences on Physical Activity in Child and Adolescent Cancer Survivors. Journal of Pediatric Psychology, 2012. 37(2): p. 198-208. 
18. Amireault, S., et al., The use of the Godin-Shephard Leisure-Time Physical Activity Questionnaire in oncology research: a systematic review. BMC Medical Research Methodology, 2015. 15(1): p. 1-11.

19. Resnick, B. and L.S. Jenkins, Testing the reliability and validity of the Self-Efficacy for Exercise scale. Nurs Res, 2000. 49(3): p. 154-9.

20. Knobf, M.T., D. Erdos, and S. Jeon, Healthy Sisters: A Feasibility study of a health behavior intervention for women of color breast cancer survivors. Journal of Psychosocial Oncology, 2018. 36(5): p. 597-608.

21. Rossi, A., et al., Feasibility of a physical activity intervention for obese, socioculturally diverse endometrial cancer survivors. Gynecologic Oncology, 2016. 142(2): p. 304-310.

22. Godin, G., J. Jobin, and J. Bouillon, Assessment of leisure time exercise behavior by selfreport: a concurrent validity study. Can J Public Health, 1986. 77(5): p. 359-62.

23. Fern, L.A., et al., The art of age-appropriate care: reflecting on a conceptual model of the cancer experience for teenagers and young adults. Cancer Nurs, 2013. 36(5): p. E27-38.

24. Kent, E.E., et al., Talking About Cancer and Meeting Peer Survivors: Social Information Needs of Adolescents and Young Adults Diagnosed with Cancer. Journal of Adolescent and Young Adult Oncology, 2013. 2(2): p. 44-52.

25. Zebrack, B.J., Psychological, social, and behavioral issues for young adults with cancer. Cancer, 2011. 117(10 Suppl): p. 2289-94.

26. Patterson, P., et al., Emerging issues among adolescent and young adult cancer survivors. Semin Oncol Nurs, 2015. 31(1): p. 53-9. 
27. Caperchione, C.M., et al., A preliminary trial examining a "real world' approach for increasing physical activity among breast cancer survivors: findings from project MOVE. Bmc Cancer, 2019. 19: p. 12.

28. Mutrie, N., et al., Recruiting Hard-to-Reach Populations to Physical Activity Studies: Evidence and Experiences. Journal of Physical Activity \& Health, 2010. 7: p. S329-S331.

29. Carroll, J.K., et al., What are successful recruitment and retention strategies for underserved populations? Examining physical activity interventions in primary care and community settings. Translational Behavioral Medicine, 2011. 1(2): p. 234-251.

30. Grimmett, C., et al., Systematic review and meta-analysis of maintenance of physical activity behaviour change in cancer survivors. International Journal of Behavioral Nutrition and Physical Activity, 2019. 16(1): p. 37.

31. Strekalova, Y.A., Finding Motivation: Online Information Seeking Following Newborn Screening for Cystic Fibrosis. Qualitative Health Research, 2016. 26(9): p. 1180-1190.

32. Santa Mina, D., et al., Connecting people with cancer to physical activity and exercise programs: a pathway to create accessibility and engagement. Current Oncology; Vol 25, No 2 (2018), 2018. 
Figures.

Figure 1. The effect of Meet \& Move on participants' mood and feelings

Figure 2. Change in perceived support and self-efficacy 